\title{
Transição dos Bacharelados Interdisciplinares para a Formação em Psicologia ${ }^{1}$
}

\author{
Monica Lima \\ Universidade Federal da Bahia, BA, Brasil. \\ Cristina Moreira Jalil \\ Universidade Federal da Bahia, BA, Brasil.
}

\author{
Denise Coutinho \\ Universidade Federal da Bahia, BA, Brasil. \\ Fábio Nieto Lopez \\ Universidade Federal do Sul da Bahia, BA, Brasil
}

\begin{abstract}
Resumo: Em 2009, o Instituto de Psicologia da Universidade Federal da Bahia propõe a área de concentração Estudos da Subjetividade e do Comportamento Humano como transição dos Bacharelados Interdisciplinares (BI) para o curso de graduação em Formação de Psicólogo. São sujeitos desta investigação os 13 estudantes egressos do BI que escolheram a transição para o curso de Psicologia. O modelo teórico-metodológico é o Sistema de Signos, Significados e Práticas, que aborda o objeto nos níveis factual, narrativo e interpretativo. Resultados: não há diferença entre coeficientes de rendimento escolar dos egressos do BI e demais estudantes de Psicologia; todos ressaltam positivamente a formação interdisciplinar do BI como relevante para o curso profissionalizante de Psicologia; a maioria reconhece sua decisão de estudar Psicologia como anterior ao ingresso no BI, atribuindo a este curso o amadurecimento da decisão; a maioria tem expectativa de exercer a profissão de psicólogo, contando com a formação prévia adquirida no BI.

Palavras-chave: Formação em Psicologia, Bacharelados Interdisciplinares, Formação Universitária, Estudos Sobre a Universidade.
\end{abstract}

\section{Transition from an Interdisciplinary Bachelor Program to a Psychology Bachelor Program}

Abstract: In 2009, the Institute of Psychology-UFBA proposed the Concentration Area of
Subjectivity and Human Behavior Studies as a transition from the Interdisciplinary Bachelor
(IB) to the Psychology Bachelor. 13 IB students were chosen as research subjects. The
theoretical-methodological model adopted was the System of Signs, Meanings and Practices,
which addresses the object from actual, narrative and interpretive levels. Results: there is no
difference in school performance levels between IB and other Psychology students; all IB
students positively emphasized interdisciplinary training as relevant to the vocational course
in Psychology; most of them recognized that their desire to study Psychology existed before
entering IB, attributing to IB the maturing of their decision; the majority of them expect to work
as psychologists, relying on the prior training acquired in IB.
KeYworDs: Psychology Training, Interdisciplinary Bachelor, University Career, University Studies.

1. Este texto é uma versão de comunicação apresentada no $2^{\circ}$ Congreso ALFEPSI: construyendo una Psicología comprometida con América Latina, realizado de 23 a 26 de outubro de 2013, em Concepción, Chile. Os autores agradecem à Pró-Reitoria de Pesquisa, Criação e Inovação (PROPCI-UFBA) pelo financiamento e bolsas de iniciação científica e à Fundação de Amparo à Pesquisa do Estado da Bahia (FAPESB) pelo apoio, sob a forma de bolsas de iniciação científica. 


\title{
Transición entre el Pregrado Interdisciplinario y el Pregrado en Psicología
}

\begin{abstract}
Resumen: En 2009, el Instituto de Psicología-UFBA propuso el área de concentración en Estudios de la Subjetividad y de la Conducta Humana como alternativa para hacer la transición desde el pregrado Interdisciplinario (GI) hacia el pregrado en Psicología. 13 estudiantes provenientes del GI que eligieron esta alternativa fueron elegidos como sujetos de esta investigación. El modelo teórico-metodológico elegido fue el Sistema de Signos, Significados y Prácticas que aborda el objeto en sus niveles factual, narrativo e interpretativo. Resultados: no hay diferencia entre el rendimiento académico de los estudiantes provenientes de GI y los estudiantes de Psicología; todos los estudiantes de GI destacan positivamente la formación interdisciplinaria del GI como relevante para la formación profesional en psicología; la mayoría de ellos reconoce su decisión de estudiar psicología como anterior al curso GI, atribuyendo a este curso la maduración de tal decisión; la mayoría espera ejercer la profesión de psicólogo confiando en la formación previa adquirida en GI.

Palabras clave: Formación en Psicología, Grado Interdisciplinario, Formación Universitaria, Estudios Sobre la Universidad.
\end{abstract}

\section{Introdução}

Entre 2006 e 2009, a Universidade Federal da Bahia - UFBA concebeu e implantou parcialmente o modelo curricular de ciclos, com base em uma nova modalidade de curso de graduação: Bacharelados Interdisciplinares (BI). Aproximadamente 1.300 vagas são oferecidas por ano no Instituto de Artes, Ciências e Humanidades Professor Milton Santos (IHAC) em quatro opções de BI: Humanidades, Artes, Ciência \& Tecnologia e Saúde. As unidades da UFBA foram convidadas e estimuladas a ofertar áreas de concentração (AC), nas respectivas unidades, para os estudantes dos BIs que desejassem tanto uma complementação de estudos mais direcionada a um determinado campo do conhecimento, quanto para os que quisessem ingressar num curso de graduação profissional. Desde o primeiro momento de sua implantação como unidade universitária, em 2009, o Instituto de Psicologia propôs a Área de Concentração Estudos da Subjetividade e do Comportamento Humano (AC-ESCH), com duas finalidades: terminalidade para o BI mais direcionada para o campo geral da Psicologia, sem, contudo, ser de cunho profissionalizante; etapa propedêutica para o Curso de Progressão Linear de Formação de Psicólogo (CPL-PSICO).

Os Bacharelados Interdisciplinares e seus desdobramentos, como a oferta organizada de vagas de seus egressos para um Curso de Progressão Linear (CPL), compõem um conjunto de ações político-pedagógicas inovadoras que precisa ser submetido ao escrutínio interrogativo à altura das condições de possibilidades de sucesso que conformam tais mudanças. O Projeto Trajetórias Acadêmicas e Construção de Significados e Sentidos na Transição dos Bacharelados Interdisciplinares à Formação em Psicologia (Lima, Coutinho, Almeida-Filho, \& Sampaio, 2012) é uma tentativa de examinar em profundidade a interface IPS-IHAC, por meio da AC-ESCH. Em outras palavras, investigar como se dá o trajeto do egresso de um BI, portador de diploma superior, que viveu uma experiência mais aberta, flexível e autônoma, diante de uma outra modalidade de curso, desta vez disciplinar, sem autonomia de percurso, profissional e linear.

Sabe-se que a formação profissional não é a única (e, talvez, nem a mais importante) função da universidade. Muitas questões merecem investimento intelectual, sobretudo aquelas referentes a processos de reestruturação curricular como este vivido pelaUFBA, desde 2009. A elaboração dos projetos político-pedagógicos dos Bacharelados Interdisciplinar foi a principal resposta da UFBA à política educacional de indução do Ministério da Educação, denominado Programa de Apoio a Planos de Reestruturação e Expansão das Universidades Federais (REUNI), instituído a partir de 2007 pelo governo federal brasileiro (Brasil, 2007). Suas principais metas para cada universidade participante incluíam a exigência, em cinco anos, de elevar a taxa de conclusão para $90 \%$ e a proporção de 18 estudantes 


\section{Lima, Monica; Coutinho, Denise; Jalit, Cristina Moreira; Lopez, Fabio Nieto (2016). Bacharelado Interdisciplinar e Psicologia.}

para cada professor, no nível da graduação. Os meios para alcançar essas metas foram estabelecidos por cada IFES. Para além desses objetivos operacionais, o Programa destacava reestruturação do ensino e da arquitetura curricular, flexibilização dos percursos formativos e melhoria da qualidade nas IFES como princípios norteadores. O conjunto de diretrizes foi elencado em seis dimensões: ampliação de oferta da educação superior pública, reestruturação acadêmicocurricular, renovação pedagógica da educação superior, mobilidade intra e interinstitucional, compromisso social da instituição e suporte da pós-graduação ao desenvolvimento e aperfeiçoamento qualitativo dos cursos de graduação (Brasil, 2007).

\section{Considerações teórico-metodológicas}

Esta pesquisa se inspira no modelo teórico-metodológico do Sistema de Signos, Significados e Práticas - S/ssp (Bibeau, 1993; Bibeau, \& Corin, 1995). O S/ssp é constituído de três níveis para a abordagem de um problema de investigação: o factual, o narrativo e o interpretativo (Bibeau, 1993). No primeiro nível, o factual, parte-se da ideia de que é preciso fazer um levantamento de fatos, ações concretas e eventos significativos para os sujeitos da pesquisa. Além disso, é necessário recolher dados complementares diversos, inclusive estatísticos, relacionados ao problema. O nível narrativo diz respeito à coleta inicial de relatos espontâneos sobre a problemática e identificação de interlocutores-chave; no nosso caso, egressos do BI e estudantes de Psicologia que ingressaram diretamente pelo vestibular. Em um segundo momento, mais sistemático, concentra-se na reconstrução propriamente dita das experiências dos entrevistados que lidam cotidianamente com a problemática.

É prudente ressaltar que as narrativas são as principais unidades de análise da proposta, e não os casos propriamente ditos, marcando um outro tipo de delineamento de pesquisa diferente do Estudo de Caso, advindo da clínica médica e psicológica, ou da prática sociológica. $\mathrm{O}$ nível interpretativo implica considerar as interpretações dos sujeitos como interpretação nativa, à qual o pesquisador não pode se limitar. A hermenêutica antropológica requer a passagem da mera descrição dos fatos e modelos explicativos nativos para a elaboração de uma interpretação, ou seja, um trabalho cooperativo analítico que propicie a emergência de sentidos que podem escapar aos atores sociais (Bibeau, 1993).

O presente processo de pesquisa consistiu em três etapas: revisão da literatura específica sobre escolha profissional em Psicologia, a partir da base SciELO-Brasil; análise dos históricos escolares dos sujeitos; realização de entrevistas semiestruturadas sobre suas trajetórias. Neste texto, destacamos alguns resultados da investigação, realizada com os 13 egressos do BI de Humanidades, que, em 2012, matricularam-se no CPL-PSICO, tendo ou não cursado áreas de concentração, bem como as repercussões para o aproveitamento de estudos do BI no curso de Psicologia. Examinamos ainda o tempo estimado para a conclusão do CLP-PSICO.

Em primeiro lugar, fizemos a análise dos 13 históricos escolares dos egressos do BI que, tendo terminado seu curso de Bacharelado em Humanidades, matricularam-se num novo curso de graduação, neste caso, Psicologia. Esses históricos foram comparados aos históricos de 46 estudantes que haviam ingressado diretamente pelo vestibular no curso de Psicologia e eram, portanto, seus contemporâneos no CPL. Esses 46 estudantes que ingressaram pelo vestibular são aqueles que esses mesmos 13 estudantes do BI encontraram no momento em que iniciaram seu segundo curso, o CPL em Psicologia, embora os anos de entrada desses dois grupos de estudantes não coincidam. Em seguida, a partir das narrativas dos egressos, examinamos suas trajetórias acadêmicas de transição do BI para o curso de Psicologia, destacando os significados e sentidos envolvidos no processo de escolha profissional e as expectativas sobre o exercício dessa profissão. Chamamos de transição a passagem de um curso pleno para outro, em função deste novo dispositivo universitário que é a Área de Concentração, um conjunto de matérias de um curso ofertadas a outro curso. Trata-se, ainda, de um dispositivo que produz uma terminalidade própria, no BI, quanto pode servir como entrada progressiva para um novo curso profissionalizante.

Para a realização das entrevistas, elaboramos um instrumento semiestruturado, com perguntas gerais que funcionaram como disparadores dos principais tópicos a serem abordados, com o intuito de minimamente direcionar as falas. O roteiro de entrevista foi montado de modo que os entrevistados pudessem falar livremente sobre suas experiências, e esse aspecto se refletiu no resultado, visto que cada entrevista se desenvolveu de maneira singular. A estrutura do instrumento levou em consideração três aspectos: experiên- 
cias anteriores à entrada no $\mathrm{BI}$; experiências durante o BI; experiências na transição e no CPL-PSICO.

\section{Sobre a AC-ESCH e o CPL-PSICO}

A estrutura curricular do BI (primeiro ciclo) prevê duas etapas de formação: Etapa Geral - com três eixos: a) Linguagens; b) Eixo Interdisciplinar. Estudos da Contemporaneidade; Estudos nas Três Culturas: Humanidades, Artes, Ciências; c) Eixo Integrador: Atividades Complementares; Etapa Específica - também com três eixos: a) Orientação Profissional: apresentação de campos / áreas profissionais; b) Área de Concentração: conjunto de estudos com coerência interna e "a serviço da construção de um perfil acadêmico e/ou ocupacional que se esgota em si mesmo" (UFBA, 2008, p. 32-33), formadas por um conjunto de Componentes Curriculares (CCs) provenientes das diversas unidades que oferecem cursos profissionalizantes específicos, antecipando os semestres iniciais de um ciclo profissional; c) Eixo Integrador: Atividades Complementares. De acordo com o Projeto Político-Pedagógico dos BIs, a área de concentração "não se define a partir da mera antecipação dos estudos básicos de carreiras profissionais e acadêmicas, o que não exclui o cumprimento da função propedêutica de etapa inicial de estudos posteriores" (UFBA, 2008, p. 32-33).

Estudos da Subjetividade e do Comportamento Humano (ESCH) é uma área de concentração oferecida pelo Instituto de Psicologia ao IHAC. Sua proposta pretende conferir a esses estudantes um domínio de conhecimentos, de caráter não profissional, concernentes ao campo da Psicologia. Apesar de oferecer uma organização curricular disciplinar, a AC busca manter a dimensão interdisciplinar da matriz e se integra ao conjunto de práticas pedagógicas em sintonia com a proposta dos BIs (Lima, \& Coutinho, 2010).

O caráter interdisciplinar da Psicologia permite receber nesta AC estudantes dos quatro BIs "pois a Psicologia, como Ciência está formalmente localizada tanto na área da Saúde quanto na área das Humanidades. Ademais, tem com o campo das Artes relações tradicionais de pesquisa e práticas de trabalho e intervenção" (Lima, \& Coutinho, 2010). O projeto de AC-ESCH foi desenhado em perspectiva aberta, em concordância com os princípios básicos do Regimento Geral da UFBA: flexibilidade, autonomia, articulação e atualização. Cada estudante pode escolher a maior parte das disciplinas de seu interesse, em trajetórias singularizadas.

Com o objetivo de manter a dimensão interdisciplinar fundamental para o BI, a AC-ESCH se estrutura com apenas dois CCs obrigatórios: "Psicologia, Ciência \& Profissão" (68h) e "Fundamentos Epistemológicos da Psicologia” (68h). Todos os CCs obrigatórios do CPL-PSICO até o $5^{\circ}$ semestre, e de acordo com a oferta semestral do curso, são progressivamente ofertados como CCs optativos ou de livre escolha. Tais CCs permitem uma aproximação compreensiva e aprofundada sobre subjetividade, representações e comportamento dos seres humanos, relevantes para práticas profissionais diversas.

Estão excluídos desta oferta CCs de conteúdo profissionalizante do curso de Psicologia. Contudo, vários estudantes têm acessado alguns desses CCs, aparentemente sem prejuízo. A integralização da AC-ESCH se dá com $1.200 \mathrm{~h}$, distribuídas em três semestres. Os CCs obrigatórios perfazem $136 \mathrm{~h}$ e, somados aos CCs optativos do Curso de Psicologia, totalizam Carga Horária $(\mathrm{CH})$ mínima de $612 \mathrm{~h}$. As demais $408 \mathrm{~h}$ são distribuídas em CCs de livre escolha, inclusive aqueles obrigatórios do próprio CPL-PSICO, num subtotal de $1.020 \mathrm{~h}$. As $180 \mathrm{~h}$ restantes são cumpridas em Atividades Complementares, podendo ser antecipadas nos três semestres iniciais do BI.

O Curso de Progressão Linear de Psicologia da UFBA (CPL-PSICO) foi criado em 1968. Ao longo das décadas seguintes, foram realizados ajustes para atualização do seu projeto pedagógico. Buscando adequar-se às Diretrizes Curriculares Nacionais para a graduação em Psicologia (CNE/CES, 2004), o curso de Psicologia da UFBA passou por ampla reforma curricular, em 2009, no momento em que este curso deixava de ser um departamento da Faculdade de Filosofia e Ciências Humanas e passa a constituir unidade independente, o Instituto de Psicologia (IPS). O atual currículo organiza-se em um Núcleo Comum, a partir de eixos estruturantes, nos sete primeiros semestres, e Ênfases Curriculares, a serem cumpridas nos três últimos semestres. O estudante pode escolher uma das duas ênfases: "Psicologia e Atenção à Saúde" e "Psicologia e Processos de Gestão". A matriz curricular vigente, desde então, apresenta diversidade de CCs com CH total de $4.216 \mathrm{~h}$, sendo $2.108 \mathrm{~h}$ de CC obrigatórios, $680 \mathrm{~h}$ de optativos e $763 \mathrm{~h}$ destinadas aos estágios, além da obrigatoriedade de cumprir $102 \mathrm{~h}$ 


\section{Lima, Monica; Coutinho, Denise; Jalit, Cristina Moreira; Lopez, Fabio Nieto (2016).}

Bacharelado Interdisciplinar e Psicologia.

de atividades complementares para a integralização curricular (Universidade Federal da Bahia, 2009).

Atualmente, no Brasil, os cursos de graduação em Psicologia formam psicólogos e licenciados em Psicologia, sendo que a primeira dimensão da formação é condição para a licenciatura, considerada complementar (CNE/CES, no 5, 2011). A Formação de Psicólogo é de, no mínimo, quatro mil horas de duração, distribuída em cinco anos (CNE/CES no 8, 2007), sendo que $15 \%$ da carga horária total do curso, obrigatoriamente, deve ser oferecida em formatado de estágio supervisionado (CNE/CES, $\left.\mathrm{n}^{\circ} 5,2011\right)$. No caso da Licenciatura em Psicologia, deverão ser acrescidas mais $800 \mathrm{~h}$, em projeto complementar ao de Formação de Psicólogo, sendo 300h obrigatórias para o estágio supervisionado (CNE/CES, ${ }^{\circ} 5,2011$ ). O CPL-PSICO da UFBA ainda não oferece Licenciatura.

O projeto pedagógico da AC-ESCH oferta em torno de 30 CCs do Curso de Psicologia aos BIs como CCs optativos. Partindo dessa ampla possibilidade de acesso, o estudante de qualquer dos quatro BIs pode completar aproximadamente $35 \%$ da $\mathrm{CH}$ total do CPL-PSICO ainda na AC-ESCH. Tais CCs ofertados aos $\mathrm{BI}$ estão inseridos na matriz curricular entre o $1^{\circ} \mathrm{e} o 5^{\circ}$ semestres de Psicologia, período em que o estudante cumpre o conjunto de CC do Núcleo Comum, sem conteúdo propriamente profissionalizante.

\section{Trajetórias acadêmicas na transição do Bacharelado Interdisciplinar para o CPL-PSICO}

Em 2012, 13 egressos (EG) do BI de Humanidades entraram no CPL-PSICO, após concluir o BI em três anos, tempo mínimo para integralização nesta modalidade. De acordo com suas fichas cadastrais, $69,2 \%$ deles são do sexo feminino; $69,2 \%$ tinham de 18 a 25 anos, ao ingressar no BI, em 2009 (faixa etária entre 18 a 46 anos); $69,2 \%$ fizeram o Ensino Médio em escola particular e 84,6\% são solteiros (Tabela 1).

Por sua vez, tomando como base os dados cadastrais dos estudantes que entraram diretamente pelo vestibular para Psicologia (EP), encontramos o seguinte perfil: idades que variam de 18 a 51 anos, sendo que $82,6 \%$ está entre 18 e 25 anos; $80,4 \%$ é do sexo feminino; $52,1 \%$ cursou o Ensino Médio em escola pública; $39,1 \%$ frequentou escolas particulares; 8,8\% não informou onde completou o Ensino Médio. Dos 46 estudantes, apenas um se declara casado e $97,8 \%$ é solteiro (Tabela 1 ).

Os egressos do BI (EG) são relativamente mais velhos do que os que ingressaram diretamente pelo vestibular (EP), o que explica o maior número de pessoas casadas naquele grupo; a disparidade entre gêneros não é tão acentuada quanto nos cursos tradicionais de Psicologia e há de fato uma diferença signi-

Tabela 1

Caracterização sociodemográfica de estudantes egressos do BI no CPL-PSICO e estudantes do CPL-PSICO que ingressaram pelo vestibular.

\begin{tabular}{lcc}
\hline \multirow{2}{*}{ Características sociodemográficas } & CPL-PSICO (EP) & Egressos BI (EG) \\
\cline { 2 - 3 } & $\mathbf{\%}$ & $\mathbf{\%}$ \\
\hline Faixa etária & \multicolumn{2}{c}{69,2} \\
$\quad$ Menos de 25 anos & & \\
Sexo & 19,6 & 30,8 \\
$\quad$ Masculino & 80,4 & 69,2 \\
$\quad$ Feminino & & \\
Tipo de escola no Ensino Médio & 52,1 & 30,8 \\
$\quad$ Pública & 47,9 & 69,2 \\
$\quad$ Privada & \multicolumn{2}{|}{} \\
Situação conjugal & 97,8 & 84,6 \\
$\quad$ Solteiro & 2,2 & 15,4 \\
$\quad$ Casado & \multicolumn{2}{|c}{} \\
\hline
\end{tabular}

CPL-PSICO: Curso de Progressão Linear de Formação de Psicólogo; EP: alunos que entraram diretamente pelo vestibular para Psicologia; BI: Bacharelado Interdisciplinar; EG: egressos. 
ficativa entre aqueles que cursaram o Ensino Médio em escola pública e os que estudaram em escolas privadas. Este dado parece contradizer o esperado, pois a suposição de que o temor pelo Exame Vestibular faria tcom que majoritariamente estudantes de escola pública acessassem o CPL-PSICO pelo BI não se confirmou neste grupo. Pelo contrário, encontramos uma proporção levemente maior de estudantes do CPL-PSICO provenientes de escola pública e, por outro lado, uma predominância de estudantes que cursaram o Ensino Médio em escolas privadas entre aqueles que ingressaram em Psicologia através do BI (Tabela 1).

No estudo intitulado "Quem é o estudante de Psicologia do Brasil?", considerando o perfil sociodemográfico dos estudantes que fizeram Exame Nacional de Desempenho (ENADE), em 2006 (total de 23.613 estudantes de Psicologia, 12.940 ingressantes e 10.673 concluintes, distribuídos em 294 cursos), $83,7 \%$ estavam em IES privadas e apenas $16,3 \%$ na rede pública (Yamamoto, Falcão, \& Seixas, 2011).

O referido estudo aponta $83,1 \%$ estudantes do sexo feminino e somente $15,9 \%$ do sexo masculino, e $73,2 \%$ do total se declaram brancos. A maioria é jovem, $31,4 \%$ dos estudantes estavam na faixa etária de 16 a 21 anos, $41,3 \%$ na faixa etária de 22 a 27 anos e $27,3 \%$ entre 28 a 78 anos. Os autores destacam que $43 \%$ estudantes estudavam no turno noturno e $49,9 \%$ no diurno, sendo que ainda $6,8 \%$ estudavam nos turnos diurno e noturno (Yamamoto et al., 2011). Tais dados sugerem que: a profissão é exercida por jovens adultos; há desigualdade de oportunidades para o ingresso no Ensino Superior em relação à etnia; a profissão é escolhida precocemente, seguindo o padrão preponderante no Brasil. Entre os egressos do BI (EG), notamos percursos que nos levaram a categorizar três tipos de trajetórias acadêmicas:

- Trajetória acadêmica 1 (TA/AC-ESCH) - cursaram a Área de Concentração Estudos da Subjetividade e do Comportamento Humano (AC-ESCH) durante o BI (10 dos 13 estudantes).

- Trajetória acadêmica 2 (TA/AC-ESCH+) - passagem pela AC-ESCH e por curso superior dentro ou fora da UFBA antes de ingressar no BI (uma estudante havia feito parte do Curso de Psicologia em outra instituição de Ensino Superior).
- Trajetória acadêmica 3

(TA/Sem AC-GA) - passagem por qualquer outra AC que não AC-ESCH ou permanência na Grande Área Humanidades (um estudante cumpriu a AC Políticas e Gestão da Cultura e outro fez a Grande Área Humanidades.

Na matriz curricular do CPL-PSICO, é esperado que o estudante que ingressa diretamente pelo vestibular (EP) cumpra, nos três primeiros semestres, $29,5 \%$ da $\mathrm{CH}$ total, pois são oferecidos seis CCs por semestre, cada um deles com $68 \mathrm{~h}$, totalizando $1.224 \mathrm{~h}$.

Na Trajetória Acadêmica 1 (TA/AC-ESCH), realizada pela maioria dos egressos (79,9\%), o aproveitamento de CCs do BI no curso de Psicologia varia de $17,4 \%$ a 28,9\%, embora os percursos sejam diversos (Gráfico 01). A carga horária total do CPL-PSICO é 4.216h.

Considerando a TA/AC-ESCH, metade dos egressos cumpriram todos os CCs relativos ao primeiro semestre da matriz curricular do CPL-PSICO; uma menor proporção (30\%) cumpriu $83,3 \%$; os demais $(20 \%)$ cumpriram 66,6\% dos CCs esperados. Em nenhum dos percursos há registro de cumprimento de $100 \%$ dos CCs, exceto os relativos ao primeiro semestre (Figura 1).

O cumprimento da carga horária de CCs optativos, previsto na matriz curricular do CPL-PSICO, é de 204h nos primeiros sete semestres. Na TA/AC-ESCH,

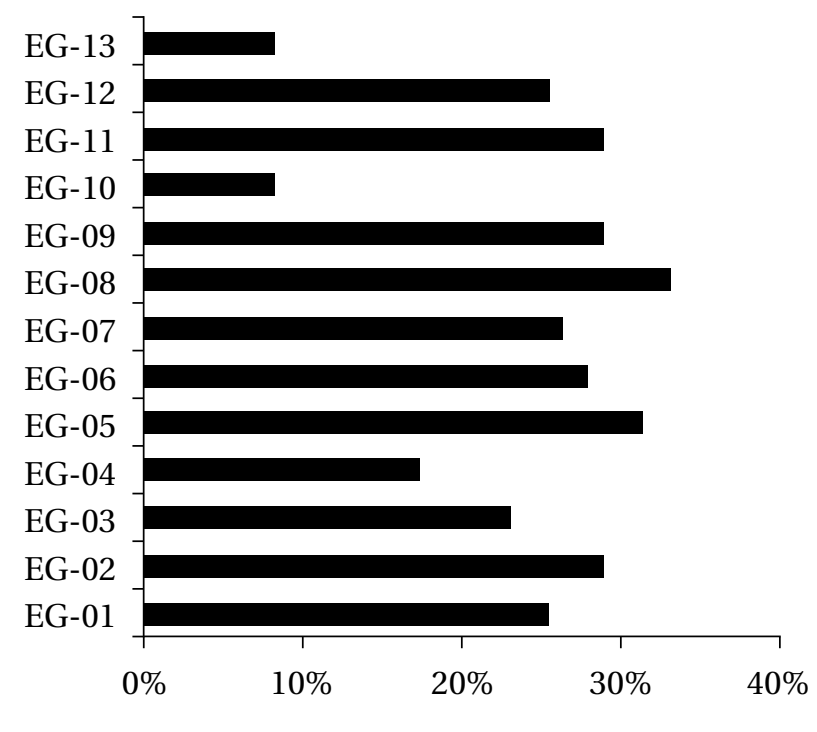

- Percentual da carga horária total

Figura 1. Distribuição de $\mathrm{CH}$ por egresso e aproveitada no CLP-PSICO, em percentual. 


\section{Lima, Monica; Coutinho, Denise; Jalil, Cristina Moreira; Lopez, Fabio Nieto (2016). Bacharelado Interdisciplinar e Psicologia.}

todos cursaram ao menos um deles, embora apenas dois tenham cumprido $100 \%$ da $\mathrm{CH}$ optativa prevista. É curioso que, com tanta $\mathrm{CH}$ cursada no BI, tão pouco possa ser aproveitado como CC optativo, se levarmos em conta a dita interdisciplinaridade do campo da Psicologia e o fato de todos eles terem vindo da área de Humanidades, na qual a Psicologia está inserida formalmente nesta Universidade.

Na Trajetória Acadêmica 2 (TA/AC-ESCH+) representada particularmente pelo percurso da EG-08, temos a maior porcentagem de aproveitamento: 33,0\% do total da $\mathrm{CH}$ necessária para integralização curricular no CPL-PSICO. Nesse particular, das $1.360 \mathrm{~h}$ equivalentes ao CPL-PSICO, 340h foram cursadas em Psicologia num outra IES (particular). Assim, a porcentagem de aproveitamento de estudos diretamente realizados no BI diminui para 24,7\% (Gráfico 1).

O aproveitamento prévio do EG-08 não incidiu sobre a diminuição de tempo de integralização curricular no BI de Humanidades. De modo singular, o estudante integralizou o referido curso com o total de $3.536 \mathrm{~h}$, valor muito superior ao mínimo estabelecido no projeto pedagógico daquele BI que é de $2.400 \mathrm{~h}$, correspondendo a $47,3 \%$ a mais de $\mathrm{CH}$. Neste caso, houve claro trajeto interdisciplinar, seguido por mais dois egressos (EG-03 e EG-11), com 39,9\% e $41,4 \%$, respectivamente (Tabela 2 ).

Na Trajetória Acadêmica 3 (TA/Sem-AC-GA), os percursos EG-10 e EG-13 mostram aproveitamento de $8,2 \%$ da carga horária do BI no CPL-PSICO (Gráfico 1), valor muito baixo em relação aos demais percursos. Nessa trajetória, os estudantes também não cursaram CCs muito além das horas de integralização mínima, seguindo a tendência da maioria dos egressos (Tabela 2), independentemente de serem ou não vinculados à AC-ESCH.
Em relação aos percursos dos estudantes que ingressaram diretamente pelo vestibular (EP) no CPL-PSICO, podemos identificar: 1) trajetória acadêmica semestralizada (TA/S), ou seja, aqueles que cumpriram no mínimo todos os CCs previstos para os três primeiros semestres $(1.224 \mathrm{~h})$, que corresponde a $73,9 \%$; 2) trajetória acadêmica dessemestralizada (TA/D), ou seja, aqueles que cumpriram menos que a carga horária esperada ou aproveitaram estudos de outra IES ou da própria UFBA, corresponde a $13 \%$; 3 ) trajetória acadêmica interrompida (TA/I), por abandono do curso ou da universidade, entre outros, corresponde a $13,1 \%$ (Tabela 3 ).

Em relação aos egressos do BI, podemos perceber uma tendência diferente. Se considerarmos o aproveitamento de estudos dos CCs cursados no BI, apenas $15,3 \%$ cumprem $\mathrm{CH}$ maior ou igual a 1.224 horas; a maioria $(61,8 \%)$ aproveita entre 816 a 1.224 horas; e $22,9 \%$ aproveitam menos de $816 \mathrm{~h}$ (Tabela 4). No entanto, a dessemestralização dos egressos do BI é consequência da dificuldade de conseguir vaga nos CCs, por não haver mecanismo institucional que lhes garanta acesso a tais CCs.

Todos os egressos do BI na transição para o CPL-PSICO são dessemestralizados, se considerarmos a lógica linear que preside o curso de Psicologia. Por outro lado, muitos estudantes que entraram diretamente pelo vestibular (EP) para o CPL-PSICO, por motivos diversos, não seguem a matriz curricular planejada, ou seja, tendem à dessemestralização (Tabela 3). Neste último caso, não se aplicam dois dos problemas operacionais que mais contribuem para a dessemestralização dos egressos do BI: falta de vaga no CC e choque de horários entre CC.

De modo geral, o coeficiente de rendimento (CR) dos egressos do BI e dos estudantes do CPL-PSICO são

Tabela 2

Diferença entre CH integralizada e a exigida para formação no BI, em porcentagem.

\begin{tabular}{|c|c|c|c|c|c|c|c|c|c|c|c|c|c|}
\hline Egressos & EG-01 & EG-02 & EG-03 & EG-04 & EG-05 & EG-06 & EG-07 & EG-08 & EG-09 & EG-10 & EG-11 & EG-12 & EG-13 \\
\hline $\begin{array}{l}\text { Carga Horária } \\
\text { Integralizada }\end{array}$ & 2.475 & 2.623 & 3.359 & 2.482 & 2.563 & 2.563 & 2.551 & 3.536 & 2.519 & 2.509 & 3.394 & 2.500 & 2.614 \\
\hline $\begin{array}{l}\text { * Diferença entre } \\
\text { a carga horária } \\
\text { integralizada e a } \\
\text { exigida }\end{array}$ & 3,1 & 9,2 & 39,9 & 3,4 & 6,7 & 6,7 & 6,2 & 47,3 & 4,9 & 4,5 & 41,4 & 4,1 & 8,9 \\
\hline
\end{tabular}


muito próximos à média geral no curso, respectivamente, 8,0 e 8,2 (Tabela 5); mas o CR de estudantes em processo de abandono do curso ou da Universidade foi excluído do cálculo. Não há, portanto, diferença em relação ao processo avaliativo, em termos de notas.

Tabela 3

Distribuição de carga horária por estudante CPL-PSICO (não BI) nos três primeiros semestres do curso, por porcentagem.

\begin{tabular}{lcc}
\hline $\begin{array}{l}\text { Carga Horária } \\
\text { Cursada }\end{array}$ & $\begin{array}{c}\text { Quantidade de } \\
\text { Estudantes (EP) }\end{array}$ & \% \\
\hline $\mathrm{CH} \geq 1224$ & 34 & 7,39 \\
$816<\mathrm{CH}<1224$ & 3 & 6,5 \\
$408<\mathrm{CH} \leq 816$ & 3 & 6,5 \\
$\mathrm{CH} \leq 408$ & 5 & 13,1 \\
\hline
\end{tabular}

CPL-PSICO: Curso de Progressão Linear de Formação de Psicólogo; BI: Bacharelado Interdisciplinar; EP: alunos que entraram diretamente pelo vestibular para Psicologia; $\mathrm{CH}$ : carga horária.

Tabela 4

Distribuição de estudantes por carga horária nos três primeiros semestres do CPL-PSICO.

\begin{tabular}{lcc}
\hline $\begin{array}{l}\text { Carga Horária } \\
\text { Cursada }\end{array}$ & $\begin{array}{c}\text { Quantidade de } \\
\text { Estudantes (EG) }\end{array}$ & \% \\
\hline $\mathrm{CH} \geq 1224$ & 2 & 15,3 \\
$816<\mathrm{CH}<1224$ & 8 & 61,8 \\
$408<\mathrm{CH} \leq 816$ & 1 & 7,6 \\
$\mathrm{CH} \leq 408$ & 2 & 15,3 \\
\hline
\end{tabular}

CPL-PSICO: Curso de Progressão Linear de Formação de Psicólogo; EG: egressos; $\mathrm{CH}$ : carga horária.

Tabela 5

Coeficiente de Rendimento de estudantes do $C P L-P S I C O$ nos três primeiros semestres e dos egressos do BI no CPL-PSICO.

\begin{tabular}{lcc}
\hline $\begin{array}{l}\text { Coeficientes de } \\
\text { Rendimento válidos }\end{array}$ & $\begin{array}{c}\text { Egressos } \\
\text { do BI }\end{array}$ & $\begin{array}{c}\text { Estudantes do } \\
\text { CPL-PSICO }\end{array}$ \\
\hline $\begin{array}{l}\text { Média dos Coeficientes } \\
\text { de Rendimento válidos }\end{array}$ & 8 & 8,2 \\
\hline
\end{tabular}

CPL-PSICO: Curso de Progressão Linear de Formação de Psicólogo; BI: Bacharelado Interdisciplinar.
No CPL-PSICO, o tempo mínimo de integralização curricular para aqueles que não fizeram BI ou que não vieram com aproveitamento de estudos de outra IES é cinco anos. No entanto, há alguns poucos estudantes que integralizam o curso em quatro anos e meio. Um dos fatores que contribui para diminuir o tempo de integralização é o aproveitamento de carga horária realizada concomitantemente ao cumprimento de CCs obrigatórios: atividades de pesquisa (participação em algum dos programas de iniciação científica) e/ou extensão (Psico-Júnior e outros), participação do curso em políticas de formação, por indução governamental (PET-Saúde e Pró-Saúde, por exemplo), que podem ser convertidas em carga horária optativa.

Fior e Mercuri (2009) destacam o mérito das atividades não obrigatórias para os estudantes, reconhecendo seu impacto positivo na formação. Partindo de relatos de experiências concretas dos seus entrevistados, as autoras concluem:

\footnotetext{
[...] no que se refere às atividades não obrigatórias, a importância atribuída ao próprio componente optativo, que possibilita o exercício da escolha por parte do aluno, incita questionamentos sobre a adequabilidade de políticas educacionais que almejam tornar obrigatórias todas as atividades desenvolvidas pelos estudantes (p. 207-208).
}

De acordo com a matriz curricular do curso de Psicologia-UFBA, o estudante deve cumprir em média seis CCs por semestre, distribuídos em cinco anos, totalizando 57 CCs. Desse modo, o tempo estimado para integralização do CPL-PSICO no caso de estudantes que vêm do BI pode variar de 2,5 a 4,0 anos. Há, no entanto, pequenas variações dependendo dos percursos construídos por cada um dos egressos. No caso da TA/AC-ESCH, pode variar de 3,0 a 3,5 anos. Particularmente na TA/AC-ESCH+, o CPL Psicologia pode ser cumprido em 2,5 anos. No entanto, nas TA/Sem AC-GA, o egresso precisará de, no mínimo, quatro anos para integralização curricular (Tabela 6).

A maioria dos estudantes que ingressou diretamente pelo vestibular (EP) para o CPL-PSICO, em 2010-2, precisará de cinco anos para integralização do curso, podendo finalizá-lo em 4,5 anos. Entretanto, podemos vislumbrar percursos que podem estender-se para além dos cinco anos, tanto quanto ocorre com estudantes que entram diretamente no 


\section{Lima, Monica; Coutinho, Denise; Jalit, Cristina Moreira; Lopez, Fabio Nieto (2016).}

Bacharelado Interdisciplinar e Psicologia.

Tabela 6

Tempo estimado em anos para integralização curricular de egressos do Bacharelado Interdisciplinar no Curso de Psicologia.

\begin{tabular}{lcc}
\hline Trajetórias & $\begin{array}{c}\text { Média de CCs necessários para } \\
\text { integralização do CPL-PSICO }\end{array}$ & $\begin{array}{c}\text { Média do tempo estimado de } \\
\text { integralização curricular }\end{array}$ \\
\hline TA/AC-ESCH & 33,6 & 3 \\
TA/AC-ESCH+ & 27 & 2,5 \\
TA/Sem AC-GA & 48,5 & 4 \\
\hline
\end{tabular}

CCs: Componentes Curriculares; CPL-PSICO: Curso de Progressão Linear de Formação de Psicólogo; TA/AC-ESCH: Trajetória acadêmica 1; TA/AC-ESCH+: Trajetória acadêmica 2; TA/Sem AC-GA: Trajetória acadêmica 3.

CPL. A exceção para menos é o EP-09, que cumpriu 32 CCs durante o BI. Nesse caso, faltava que o estudante cursasse apenas 25 CCs. Para integralização do seu curso, ele precisa idealmente de apenas dois anos em Psicologia. A análise do tempo de integralização curricular para os EG cumprirem o CPL-PSICO carece de uma árdua estratégia de coleta de dados para apuração mais precisa dessa estimativa, que não foi incluída no desenho metodológico do presente estudo. Necessitaríamos dispor de dados ainda não disponíveis no sistema acadêmico da UFBA, que permitissem calcular as médias do tempo de integralização curricular dos estudantes que ingressaram diretamente pelo vestibular para o CPL-PSICO para servir de parâmetro comparativo.

\section{Escolha profissional dos egressos do BI no curso de Psicologia: contribuições do BI ao processo de formação profissional}

Em geral, escolhas profissionais feitas antes da entrada na universidade são, na melhor das hipóteses, idealizações, projeções construídas a partir de experiências de pais e demais familiares, amigos, professores e outros profissionais que eventualmente estão presentes na vida desses jovens. Podem ser diversas as motivações para a escolha de uma profissão, dentre elas, a continuidade da carreira dos pais, o interesse por uma área do conhecimento, status e inserção no mundo do trabalho (Ojeda, Creutzberg, Feoli, Melo, \& Corbellini, 2009; Barreto, \& Aiello-Vaisberg, 2007).

Ao iniciar um curso em uma universidade, geralmente o estudante possui apenas uma visão geral e estereotipada sobre a profissão e o curso que escolheu (Costa, 2007; Ojeda et al., 2009). Muitas vezes, segue cheio de dúvidas quanto à escolha e, ao adentrar a graduação, depara-se com currículos rígidos e pouca - por vezes, nenhuma - possibilidade de troca, transição e mudança entre cursos, ou mesmo alternativas e flexibilidade reduzidas dentro da carreira escolhida. Esse é um dos aspectos relacionados às grandes taxas de evasão nos primeiros anos da graduação. Do mesmo modo, adaptações à vida em uma nova cidade, a um modelo de vida estudantil bastante diverso daquele experimentado nos Ensino Fundamental e Médio são, dentre outros, fatores que podem contribuir para a chamada "evasão em um curso universitário" (Bueno, 1993; Coulon, 2008). Alguns estudos apontam, ainda, entre concluintes e graduados em cursos de progressão linear aspectos recorrentes como falta de preparo para o ingresso no mundo do trabalho, dificuldades na definição de projetos de carreira e quanto aos papéis esperados dentro da profissão pretendida (Dias, \& Soares, 2010; Lemos, Bueno, Silva, \& Genicolo, 2007).

Quando entrevistados sobre suas trajetórias acadêmicas desde a primeira entrada na UFBA, através do BI, e posterior ingresso no CPL-PSICO, os sujeitos desta investigação foram incentivados a abordar alguns aspectos apontados na literatura sobre processo de escolha profissional e expectativa de exercer a profissão. Nesta direção, exploramos algumas temáticas que tangenciam o processo: a) contato anterior com a Psicologia; b) motivações para a entrada no BI; c) decisão de vincular-se à AC-ESCH; d) contribuições do BI para a formação universitária e decisão pela Psicologia.

Os entrevistados não demonstram muita clareza sobre motivos que os fizeram optar pela formação em Psicologia. Quando provocados a explorar o tema, atribuem sua vontade de fazer o curso a determinadas características pessoais ou que julgavam ser típicas de psicólogos, como: "ser bom ouvinte"; "gostar de compreender as pessoas"; "ouvir mais que falar"; "costume de escutar os amigos", entre outros. Todos 
esses exemplos compõem o estereótipo desse profissional no imaginário social.

Em relação ao contato com a Psicologia ou com psicólogos, antes da entrada na universidade, relembram o despertar do interesse a partir de: contatos com amigo, parente, estudante ou profissional de Psicologia; testes vocacionais (realizados inclusive por pedagogos). Com ou sem contato prévio com agentes do campo da Psicologia, alguns relatam "vontade de fazer Psicologia desde cedo", por vocação, "tino" e “jeito" para exercer algo, sem muita clareza do que seria Psicologia. Enfatizam, porém, desejo ou afeto intensos. Alguns localizaram o momento da escolha apenas quando da apresentação da AC-ESCH, já no BI.

As motivações para entrar na UFBA através do BI são relatadas de modos singulares, mas podemos perceber que alguns significados são compartilhados por boa parte dos sujeitos entrevistados. Alguns relataram a certeza de que desejavam cursar Psicologia, já tendo feito vestibular anteriormente sem êxito, ou até cursado uma graduação completa em outro curso superior; para outros, a dúvida entre Psicologia e outro curso foi uma constante. Para os entrevistados mais jovens e sem curso superior prévio à entrada no BI, a dúvida é mais contundente. Percebemos, no entanto, que cursar o BI está relacionado a alguns significados positivos, por exemplo, "possibilidade de fazer a escolha mais consciente e segura". Somado a este aspecto, e fortalecidos com a vivência no BI, torna-se mais fácil fazer a escolha de um curso muito concorrido, como é o caso da Psicologia, que há muitos anos figura entre os três cursos mais disputados da UFBA.

Alguns dos egressos entrevistados julgavam-se não preparados para enfrentar a concorrência da UFBA, ou seja, viam-se sem condições de sustentar a escolha e apostar num curso realmente bastante disputado. Estes, então, viram no BI uma alternativa possível, inclusive para voltar a estudar, vindo a desenvolver curiosidade pela proposta interdisciplinar. Principalmente para aqueles que fizeram a AC-ESCH, a experiência do BI proporcionou um período de maturação da decisão de fazer Psicologia. O contato com o leque de CCs da Psicologia possibilitou conhecimento da diversidade teórico-profissional do campo. Para alguns, esse contato foi crucial para a escolha, já que tinham certeza ou inclinação por outro curso, por exemplo, Direito.

Para além de ajudar na escolha profissional ou realizar um desejo deixado em algum lugar do passado, as contribuições do BI presentes nessas narrativas vão muito além do que esperávamos. Os relatos são muito intensos; cursar o BI está relacionado com experimentar, amadurecer, aperfeiçoar, aprender, ter acesso a um tipo de conhecimento que "abriu a cabeça", "ampliou os horizontes", forneceu "visão crítica", possibilitando consolidação e confirmação da escolha profissional. Todos falaram sem hesitar que fariam o BI novamente antes de entrar na Psicologia, mesmo os mais jovens que se sentem um pouco "atrasados" com os três anos prévios do $\mathrm{BI}$, antes de acessar um curso profissionalizante. Para aqueles cujo aspecto temporal era muito relevante, a tendência foi circular menos pelas demais áreas de conhecimento, centrando a formação no BI em CCs prioritariamente da Psicologia, na perspectiva de integralizar o curso mais rapidamente. Embora seja a expectativa da maioria, nem todos pretendem exercer a profissão de psicólogo.

A conclusão do curso de Psicologia e a pretensão de exercer a profissão não é algo garantido. No Brasil, 53\% das(os) psicólogas(os) atuam exclusivamente na área; para $10 \%$ a Psicologia é a principal atividade, mas não a única; $11 \%$ exerce secundariamente a profissão, $15 \%$ não exerce, mas pretende exercer; $4 \%$ não exerce e não pretende exercer; $4 \%$ já exerceu no passado; $4 \%$ não exerce nenhuma atividade remunerada (Lhullier, \& Roslindo, 2013). Em resumo, $27 \%$ dos psicólogos no Brasil não exercem a profissão. Tal dado envolve muitos aspectos, mas não contamos ainda com estudos que possam esclarecê-los.

Nesse particular, há expectativa de que a passagem pelo BI e a possibilidade de aproximação prévia qualificada com a Psicologia possa ter algum impacto em relação ao investimento realizado pelo estudante e a perspectiva de exercer a profissão.

\section{Considerações finais}

Um dos pontos mais recorrentes quando se pensa na entrada de egressos do BI em um CPL diz respeito ao tempo de permanência do estudante na universidade. Para muitos, e em bases totalmente reativas, o estudante que faz tal percurso demorará muito para finalizar o curso profissionalizante, "guardando" por mais tempo vaga pública. Em que pese a falta de dados sobre o assunto no curso de graduação em Psicologia (IPS/UFBA), um olhar um pouco mais cuidadoso sobre dados em outras unidades e na universidade brasileira mostrará facilmente que não há grande dispari- 


\section{Lima, Monica; Coutinho, Denise; Jalit, Cristina Moreira; Lopez, Fabio Nieto (2016). Bacharelado Interdisciplinar e Psicologia.}

dade, comparando-se o tempo médio do estudante que ingressa pelo vestibular diretamente no CPL com aquele que ingressa no CPL por meio do BI. Como foi demonstrado nos resultados, estudantes do BI têm possibilidade, se assim desejarem, de passar pela área de concentração e ingressar no CPL-PSICO com até aproximadamente $35 \%$ da $\mathrm{CH}$ aproveitada.

No que diz respeito à capacidade dos estudantes para acompanharem CCs da Psicologia, não foi possível perceber diferença significativa em relação às médias de aprovação (notas) dos estudantes egressos do BI e aqueles que entraram por vestibular diretamente (EP) no CPL-PSICO nos mesmos CCs e semestres. É factível afirmar que tais estudantes, mesmo antes de ingressar no curso profissionalizante e após um ano e meio de formação geral, são capazes de obter aproveitamento satisfatório nos CCs aos quais tiveram acesso. Nessa direção, observamos que dois estudantes egressos do BI apresentaram reprovação por nota em um CC de Psicologia. Esse desempenho não é muito diferente do desempenho do estudante que entra pelo vestibular (EP), pois, em relação à reprovação por nota, identificamos uma ou duas reprovações nos três semestres analisados em cinco percursos distintos desses estudantes (EP). A facilidade em acompanhar os CCs foi ratificada nas entrevistas, já que, de maneira geral, egressos do BI não apontaram dificuldades significativas no que diz respeito ao acompanhamento do conteúdo dos CCs.

A análise dos históricos escolares também possibilitou que conhecêssemos percursos singulares de ingresso pelo vestibular (EP), que fogem do padrão idealizado pela matriz curricular. São trajetórias que, curiosamente, aproximam-se dos percursos dos egressos do BI. Nessa direção, entendemos que os percursos dos egressos do BI radicalizam a ideia

\section{Referências}

Barreto, M. A., \& Aiello-Vaisberg, T. (2007). Escolha profissional e dramática do viver adolescente. Psicologia e Sociedade, 19(1), 107-114. doi:10.1590/S0102-71822007000100015

Bibeau, G., \& Corin, E. (1995). From submission to the text to interpretive violence. In G. Bibeau, \& E. Corin. (Eds.), Beyond textuality. Ascetism and violence in anthropological interpretation approaches to semiotics series. Berlin: Mouton de Gruyter. de flexibilização e articulação dos currículos, permitindo que problematizemos, a partir de experiências concretas, normas, regras, concepção e organização curriculares clássicas.

No grupo de egressos do BI (EG) entrevistados, o número de estudantes que entrou no BI pensando em posteriormente migrar para o CPL de Psicologia foi pequeno. A maioria se encontrava em dúvida e identificou no BI a possibilidade de fazer uma escolha mais segura e consciente. Nesses casos, havia os que estavam divididos entre a Psicologia e outros cursos, tendo a intenção de, no BI, adquirir conhecimento maior sobre outras áreas. Cabe ressaltar que um dos egressos do BI que ingressou no curso de Psicologia também era mestrando em um Programa de Pós-Graduação na própria UFBA. Quando entrevistado, ele relatou aspectos positivos dessa trajetória singular, como estudante da graduação e da pós-graduação simultaneamente, e afirmou pretender exercer a profissão de psicólogo.

Muitos egressos se referiram à necessidade de vivenciar dentro da universidade uma etapa preparatória de escolha profissional e viram no BI a possibilidade de experimentar esse momento de amadurecimento da vivência acadêmica e, eventualmente, construir as bases para entrar num curso profissionalizante ou, como permite esta modalidade, ir diretamente para a pós-graduação ou para uma licenciatura.

Até o momento, essas são as trajetórias que conseguimos capturar. Trata-se da primeira turma de egressos do BI que faz a passagem para o curso profissionalizante de Psicologia. Muito ainda temos que percorrer no acompanhamento de novas turmas, a fim de chegarmos a resultados mais robustos sobre tal experiência que, até aqui, tem-se mostrado produtiva e proveitosa para os envolvidos.

Bibeau, G. (1993). Hay una enfermedad en las Américas? Otro camino de la antropología médica para nuestro tiempo. In C. Pinzón, P. R. Suárez, \& G. Garay. (Ed.). Cultura y aluden la construcción de las Américas (pp. 41-70). Bogotá: Instituto Colombiano de Antropología.

Brasil (2007), Ministério da Educação, Secretaria de Educação Superior. Reuni: reestruturação e expansão das universidades federais: diretrizes gerais. Brasília, DF: o autor. 
Brasil (2007, 13 de junho), Conselho Nacional de Educação - CNE. Parecer CNE/CES no 8/2007. Dispõe sobre carga horária mínima e procedimentos relativos à integralização e duração dos cursos de graduação, bacharelados, na modalidade presencial. Diário Oficial da União.

Brasil (2011, 16 de março), Conselho Nacional de Educação - CNE. Resolução CNE/CES no 5, de 15 de março de 2011. Institui as Diretrizes Curriculares Nacionais para os cursos de graduação em Psicologia, estabelecendo normas para o projeto pedagógico complementr para a Formação de Professores de Psicologia. Diário Oficial da União.

Bueno, J. L. O. (1993). A evasão de alunos. Paidéia (Ribeirão Preto), (5), 9-16. doi:10.1590/S0103-863X1993000200002

Costa, J. M. (2007). Orientação profissional: um outro olhar. Psicologia USP, 18(4), 79-87. doi:10.1590/S0103-65642007000400005

Coulon, A. (2008). A condição de estudante: a entrada na vida universitária. Salvador, BA: EDUFBA.

Dias, M.S.L., \& Soares, D.H.P. (2012). A escolhaprofissional no direcionamento da carreira dos universitários. Psicologia: Ciência e Profissão, 32(2). 272-283. doi:10.1590/S1414-98932012000200002

Fior, C., \& Mercuri, E. (2009). Formação universitária e flexibilidade curricular: importância das atividades obrigatórias e não obrigatórias. Psicologia da Educação, (29), 191-215. Recuperado de http://pepsic.bvsalud.org/scielo.php?script=sci_arttext\&pi$\mathrm{d}=$ S1414-69752009000200010\&lng=pt\&nrm=iso

Lemos, C. G., Bueno, J. M. H., Silva, P. L., \& Genicolo, V. C. (2007). Referenciais de carreira e identidade profissional em estudantes universitários. Psicologia: Ciência e Profissão, 27(2), 208-223. doi:10.1590/S1414-98932007000200004

Lhullier, L. A., \& Roslindo, J. (2013). As psicólogas brasileiras: levantando a ponta do véu. In L. A. Lhullier. (Org.), Quem é a psicóloga brasileira? mulher, psicologia e trabalho. Brasília, DF: Conselho Federal de Psicologia.

Lima, M. \& Coutinho, D. (2010). Projeto pedagógico da área de concentração em Estudos da Subjetividade e do Comportamento Humano (AC-ESCH). Sal- vador, BA: Instituto de Psicologia, Universidade Federal da Bahia.

Lima, M., Coutinho, D., Almeida-Filho, N., \& Sampaio, S. (2012). Trajetórias acadêmicas e construção de significados e sentidos na transição dos Bacharelados Interdisciplinares à formação em Psicologia na UFBA. Recuperado de https://repositorio. ufba.br/ri/bitstream/ri/5660/1/Lima\%2c\%20 et.\%20al\%2018-04-2012\%20\%28reposit\%C3\%B3rio\%29.pdf

Ojeda, B. S., Creutzberg, M., Feoli, A. M. P., Melo, D. S., \& Corbellini, V.L. (2009). Acadêmicos de enfermagem, nutrição efisioterapia:a ascolhaprofissional. Revista Latino-Americana de Enfermagem, 17(3), 396-402. doi:10.1590/S0104-11692009000300018

Universidade Federal da Bahia - UFBA (2008). Projeto pedagógico dos bacharelados interdisciplinares. Salvador. Recuperado de https://www.ufba.br/ sites/devportal.ufba.br/files/implant_reuni.pdf

Universidade Federal da Bahia (2009), Instituto de Psicologia - IPS. Projeto político pedagógico do curso de graduação em psicologia. Salvador, BA: o autor. Yamamoto, O. H., Falcão, J. T. R., \& Seixas, P. S. (2011). Quem é o estudante de psicologia do Brasil?. Avaliação Psicológica, 10(3), 209-232. Recuperado de http://pepsic. bvsalud.org/scielo.php?script=sci_arttext\&pi$\mathrm{d}=$ S1677-0471201 1000300002\&lng=pt\&nrm=iso

\section{Monica Lima}

Psicóloga, Doutora em Saúde Coletiva pela Universidade Federal da Bahia e professora do Instituto de Psicologia da Universidade Federal da Bahia, Salvador-BA. Brasil.

E-mail: molije@hotmail.com

\section{Denise Coutinho}

Psicóloga, Doutora em Letras pela Universidade Federal da Bahia e professora do Instituto de Psicologia da Universidade Federal da Bahia, Salvador - BA. Brasil. Cedida à Universidade Federal do Sul da Bahia, Itabuna-BA. Brasil.

E-mail: denisecoutinho1@gmail.com 


\section{Cristina Moreira Jalil}

Psicóloga, Mestranda no Programa de Filosofia da Universidade Federal da Bahia, Salvador - BA. Brasil.

E-mail: tinajalil@hotmail.com

\section{Fábio Nieto Lopez}

Psicólogo, Doutor em Psicologia pela Universidade

Federal da Bahia e professor da Universidade Federal

do Sul da Bahia, Itabuna - BA. Brasil.

E-mail: fabionieto@gmail.com

\section{Endereço para envio de correspondência:}

Universidade Federal da Bahia, Instituto de Psicolo-

gia, Rua Aristides Novis, 197. Campus São Lázaro.

CEP: 40210-909.

Salvador-BA. Brasil.

Recebido 17/10/2013

Aprovado 26/01/2016

Received 10/17/2013

Approved 01/26/2016

Recibido 17/10/2013

Aceptado 26/01/2016

Como citar: Jesus, M. L., Coutinho, D., Jalil, C., \& Lopez, F. (2016). Transição dos bacharelados interdisciplinares para a formação em psicologia. Psicologia: Ciência e Profissão, 36(1): 183-195. doi:10.1590/1982-3703002042013

How to Cite: Jesus, M. L., Coutinho, D., Jalil, C., \& Lopez, F. (2016). Transition from an interdisciplinary bachelor program to a psychology bachelor program. Psicologia: Ciência e Profissão, 36(1): 183-195.

doi:10.1590/1982-3703002042013

Cómo citar: Jesus, M. L., Coutinho, D., Jalil, C., \& Lopez, F. (2016). Transición entre el pregrado interdisciplinario y el pregrado en psicología. Psicologia: Ciência e Profissão, 36(1): 183-195. doi:10.1590/1982-3703002042013 